\title{
A processing method for rough cutting hourglass worm on general NC lathe
}

\author{
Xinyang QIU*, Yanqin ZHANG*, Qiwu ZHOU** and Fangyan ZHENG** \\ * College of Mechanical and Electronic Engineering, Northwest A\&F University \\ 22 Xinong Rd., Yangling District, Shaanxi Province, 712100, P. R. China \\ E-mail: zhangyanqin@nwafu.edu.cn \\ ${ }^{* *}$ Chongqing Key Laboratory of Time-Grating Sensing and Advanced Testing Technology, Chongqing University of Technology \\ 69 Hongguang Avenue, Banan District, Chongqing, 400054, P.R. China
}

Received: 15 January 2019; Revised: 27 May 2019; Accepted: 25 July 2019

\begin{abstract}
The manufacturing method of two spatial-orthogonal rotary axis linkage is usually used to cut hourglass worm. However, the traditional processing method of roughing tooth groove depends on the special machine, specific tool, and its processing efficiency is lower. To avoid these defects, a processing method of the rough cutting hourglass worm on general $\mathrm{NC}$ lathe without $\mathrm{C}$-axis functions was presented in the paper. Based on the forming principle of the hourglass worm and the traditional processing steps, the machining principle of the new approach was elaborated. Key techniques such as the shape of the blade, the contours of tooth groove, tool path loop and the variable lead of worm, etc. were discussed. Macro-program of the new method was programmed based on Fanuc oi mate-tc CNC system, and tooth groove of the hourglass worm were trial manufactured on general NC lathe. Addendum axial pitch and a normal tooth thickness of the trial model were measured. It is noted that measurement results can satisfy the technological requirement. In conclusion, the new processing method of roughing hourglass worm is feasible and efficient.
\end{abstract}

Keywords : Processing method, Hourglass worm, Roughing tooth groove, Macro programming, General NC lathe

\section{Introduction}

Hourglass worm drives have multi-teeth meshing, distribution of the instantaneous contact line nearly along the tooth-height direction, larger lubrication angular, outstanding performance in carrying capacity and other prominent advantages (Shimachi et al., 1994; Wang et al., 2002; Wang et al., 2013). They are widely used in metallurgy, mining, petroleum, marine, military, and other heavy-duty transmission applications. Manufacturing processes of hourglass worm tooth can be divided into roughing tooth groove and finishing tooth surface (Dong et al., 2012; Карпев, 1958; Zhao and Zhang, 2011). In the finishing process, special surfaces of hourglass worm tooth are shaped by the different type of generatrix. In the roughing step, similar or identical tooth grooves are cut out by turning or milling methods. However, roughing step is very important to improving the machining efficiency of worm parts, because most of the material needs to be removed and force status of cutter edge are complicated in roughing process.

Hourglass worm drives are usually used to transmit motion and power between two spatial-orthogonal rotary axes. In theory, hourglass worm tooth is manufactured based on the location and motion relationship between the hourglass worm and its worm gear. Therefore, processing machine tools of the hourglass worm should have two spatial-orthogonal rotary axes, which two axes can be operated in the linkage working condition. This approach is named generating method (Cheng, 2008; Qin, 2011; Qiu, 2011). On the one hand, it is suitable to design a fixed-special machine for middle or large batch production, but a fixed-special machine is rarely reported. On the other hand, it is appropriated to modify gear hob machine or refit lathe for single piece or small batch production. Review of references shows that generating method (Du and Jiang, 1988) is widely used to machining tooth groove of the hourglass worm. 
However, the usual roughing method relies on retrofit machine tools; the cutter should be designed for special purposes; and its processing efficiency of using the traditional roughing method to cut tooth groove is lower.

To avoid the defects of the traditional roughing method, this article proposes a new rough processing method for turning the tooth groove of the hourglass worm on general $\mathrm{NC}$ lathe. In this study, the forming principle of the hourglass worm and the processing steps will be expounded. The machining principle of the brand-new approach will be elaborated. The new method and its' key techniques such as the shape of cutting edge, the contours of tooth groove, tool path loop and the variable lead of worm, etc. will be discussed. Macro-program of the method will be programmed based on Fanuc oi mate-tc CNC system, and tooth groove of the hourglass worm will also be trial manufactured on general NC lathe.

\section{The forming principle of hourglass worm and the traditional roughing method}

\subsection{The generating theory of a common hourglass worm}

Tooth surface of the Hindley worm is a sweep surface (Li et al., 2011), which is formed by a straight line in the conjugation movement. Tooth surface of enveloping hourglass worm is enveloping surface, which is formed in the conjugated movement by curved-surfaces such as planar, taper surface, revolution surface of involute, etc. (Liu et al., 2011; Shi et al., 2004; Zhao et al., 2010). Straight line or curved-surfaces mentioned above is called the generatrix of the hourglass worm. We usually classified the hourglass worm gears according to the type of the generatrix. The conjugated movement mentioned above is also called the relative motion between worm blank and cutting tools. In typical cases, we defined the corresponding movement based on the location and motion relationship between the hourglass worm and its worm gear (Chen et al., 2016; Rui et al., 2018).

The generating theory of the planar enveloping hourglass worm (Qiu et al., 2011; Shi et al., 2016) is shown in Figure 1. The shortest distance between rotary axis $O_{1}-O_{1}$ and $O_{2}-O_{2}$ is machining center distance $a$. The crossing angle between axes $O_{1}-O_{1}$ and $O_{2}-O_{2}$ is $90^{\circ}$. The generatrix $\Sigma^{(2)}$ rotates about axis $O_{2}-O_{2}$ with the angular velocity vectors $\omega_{2}$. Meanwhile, the hourglass worm blank rotates about axis $O_{1}-O_{1}$ with $\omega_{1} . \omega_{1} / \omega_{2}$ is called machining transmission ratio, which is kept constant. The generatrix $\Sigma^{(2)}$ converts to a spatial planar cluster in the relative motion effect. Parts of the enveloping surface of the cluster intersect with the worm blank, which is one side of the tooth surface of the planar enveloping hourglass worm.

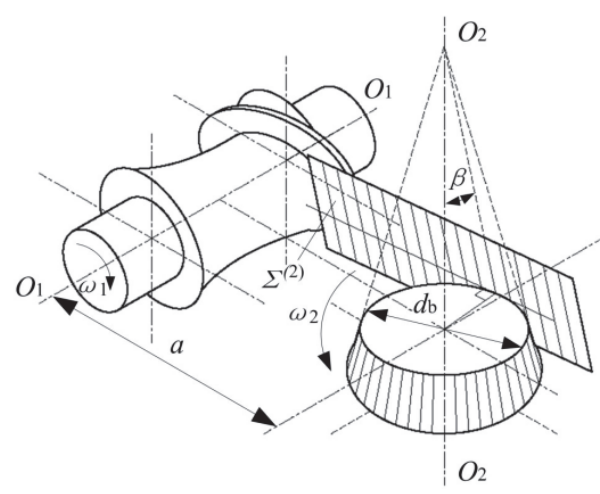

Fig.1 The generating theory of the planar envelopment hourglass worm

\subsection{Manufacturing processes of hourglass worm}

Manufacturing processes of hourglass worm tooth can be divided into roughing tooth groove and finishing tooth surface. In practical production, we mainly use the generating method to finish tooth surface processing. Therefore, machining principles of finishing step must keep insistent with generating theory of the hourglass worm. First, the main working face of the cutting tools must keep identical with the generatrix in generating theory. Second, the relative motion between worm blank and cutting tools must keep consistent with the conjugated movement mentioned in subsection 2.1 .

Roughing tooth groove should remain the necessary machining allowance for the finishing tooth surface, and contour shape of the tooth groove don't have to keep consistent with the theoretical tooth profile. Cutting edge or working face of roughing tools may have differed from the generatrix in generating theory, but the relative motion of roughing should be determined by the conjugated movement. The method is called the traditional method for roughing. 
The usual roughing method is implemented as rough-turning the Hindley worm or rough-milling conical surface enveloping hourglass worm. Tooth groove of the hourglass worm is rough processed by turning tools or milling cutter in the retrofitted machine tools of the hourglass worm.

\subsection{Essential motions of traditional roughing method}

Based on the generating theory, roughing machine has at least two rotary motion axes, one for worm blank and the other for cutting tools. Two axes are connected by a precise and adjustable drive train or controlled by CNC technology to achieve linkage, and two axes should usually remain spatial-orthogonal relationship (Du and Jiang, 1988; Карпев, 1958). By refitting vertical gear hob machine or horizontal lathe, it is easy to realize the above requirements. The program is an application of two spatial-orthogonal rotary axis linkage technology.

According to the basic principles of composition and decomposition of motions, the rotational motion of the cutting tools about the axis $\mathrm{O}_{2}-\mathrm{O}_{2}$ can be decomposed into another rotational motion and two translational motions in the median plane of the worm gear. And then considering the rotary movement of worm blank, there are four movements totally. Controlling the four movements by CNC technology, to make them work in the linkage mode, we can easily obtain virtual rotational center functions (Qiu et al., 2011; Zhang and Li, 2009). Based on a horizontal lathe, rebuilding motion axes of the spindle, transverse pallet and longitudinal pallet by CNC technology, and installing a CNC vertical rotary table on the small pallet, we can get a multifunctional machine tool for processing hourglass worm tooth. Of course, the machine can be easily used for roughing. The program is an application of four axes linkage technology.

The above method can ensure that straight cutting edge is always tangent to the main basic circle in generating processing. Therefore, trapezoidal tooth profile of roughing work piece can be formed by the cutter with a straight cutting edge.

\section{Machining principle of new roughing method}

Work piece rotates together with work spindle of ordinary horizontal NC lathe. Rotational motion is the main movement of turning. A spindle of ordinary $\mathrm{NC}$ lathe has realized speed control, which can work in the case of invariant rotational speed or constant linear velocity (Dudas et al., 2015). However, most of the spindle has not achieved position control, which does not have the $C$-axis functions, which cannot be achieved position-linkage control with other feed axes. Cutting tool translates along with two orthogonal linear axes. The translational motions are the feed movements of turning process. Two orthogonal linear axes are controlled by CNC system, which can work in a two-axis position-linkage state. Thus, the tip can translate along the fitting planar curves. The fitting planar curves are contour lines of rotational parts. However, work spindle of general $\mathrm{NC}$ lathe is usually equipped with pulse encoder. When the NC lathe is used for threading, the pulse encoder can avoid teeth disorder.

For turning toroidal helical teeth with trapezoidal tooth profile in ordinary horizontal NC lathe, we propose a new processing method. The machining principle of the brand-new roughing method is shown in Figure 2. XOZ is the interpolation plane of the NC lathe. Worm blank goes around axis $Z$ together with spindle at constant rotation speed $\omega$. Cutting point $p$ translates along the arc $l$ in the plane $X O Z$, which is interpolated by two orthogonal linear axis linkage. Different tool path loop corresponds to distinct circular line $l$. Translational velocity represents the letter $F$, which means feed per revolution. Values of $F$ equal the "pitch" at point $p$ on tools-gear. The center of circular $\operatorname{arc} l$ is $O_{0}$, and the radius of $l$ is $r_{p}$. Theoretical machining center distance is $a$. Basic circle radius of theoretic tools-gear is $r_{\mathrm{b}}$. To avoid thread teeth disorder, we must ensure that the spindle is in the same phase when the point $p$ is at the beginning of the thread teeth. 


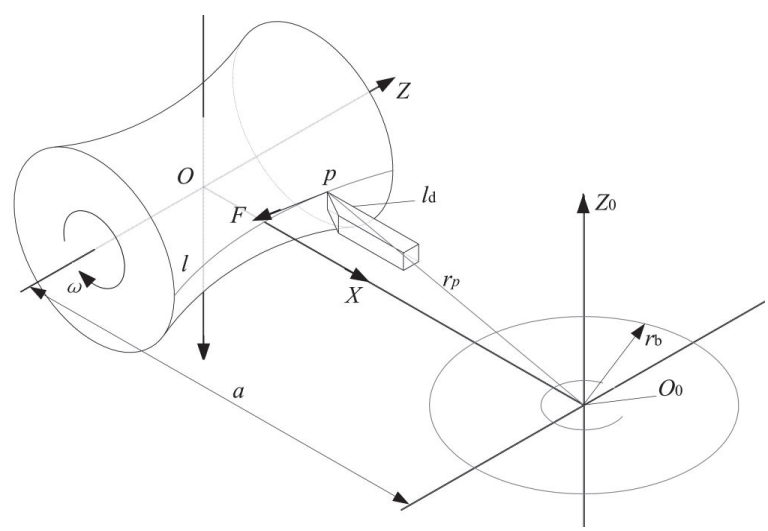

Fig. 2 The machining principle of new roughing method of hourglass worm

As shown in Figure 2, there are three coordinate axes that are controlled by CNC technology. Work spindle has realized speed control, and two orthogonal linear axes have realized position-linkage control. Obviously, two orthogonal linear axis linkage technology of brand-new roughing method is different from two spatial-orthogonal rotary axis linkage technology of the traditional roughing method. Compared with the reference (Zhang and $\mathrm{Li}$, 2009), work spindle in the new roughing method is not necessary to achieve the position control, and which lacks a rotation axis perpendicular to the plane $X O Z$. Accordingly, the new roughing method cannot ensure that "straight cutting edge" is always tangent to the main basic circle, in fact, which can only ensure that cutting point may meet the speed ratio of conjugate movement. To apply the new roughing method for machining trapezoidal tooth profile, we need to control cutting point by CNC technology, and use the locus method with multiple tool paths.

\section{Key techniques of new roughing method}

To machining toroidal helical teeth with trapezoidal tooth profile in ordinary horizontal NC lathe, we must discuss the following key issues.

\subsection{Selection of the blade shape}

From the machining principle of new roughing method, the cutting tool can only translate in the $X O Z$ plane, and it cannot turn around. When selecting the blade shape, it should avoid interference with the theoretical surface of the worm. The limitations of the trapezoidal tooth profile of the hourglass worm on the blade shape are shown in Figure 3. In the axial interface of the hourglass worm, point $p_{\mathrm{s} 1}, p_{\mathrm{s} 2} 、 p_{\mathrm{s} 3} 、 p_{\mathrm{s} 4}$ is boundary points of worm groove in the theoretical starting position, and point $p_{\mathrm{e} 1} 、 p_{\mathrm{e} 2} 、 p_{\mathrm{e} 3} 、 p_{\mathrm{e} 4}$ is boundary points of worm groove in the theoretic termination position. Tooth profile $p_{\mathrm{s} 1}-p_{\mathrm{s} 3} 、 p_{\mathrm{s} 2}-p_{\mathrm{s} 4} 、 p_{\mathrm{e} 1}-p_{\mathrm{e} 3} 、 p_{\mathrm{e} 2}-p_{\mathrm{e} 4}$ is straight line profile. To prevent over-cutting, when the cutting-point $p$ is located at the point $p_{\mathrm{s} 3}$, the right side of the blade should avoid the machined tooth profile $p_{\mathrm{s} 1}-p_{\mathrm{s} 3}$, and while the cutting point $p$ is located at the point $p_{\mathrm{e} 4}$, the left side of the blade should avoid machining the tooth profile $p_{\mathrm{e} 2}-p_{\mathrm{e} 4}$.

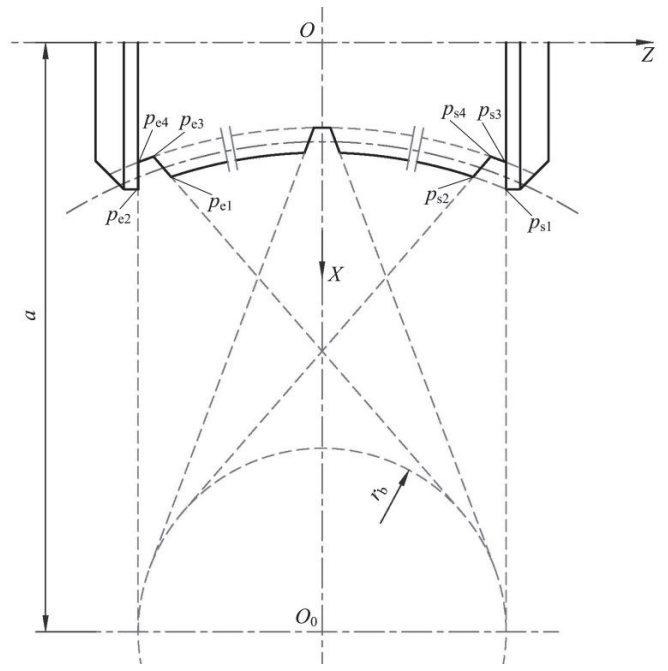

Fig. 3 Limitations of trapezoidal tooth profile on the blade shape 
Considering the blade width and the nose radius, available blade shape of the new roughing method is shown in Figure 4. The width of the blade is $b_{\mathrm{d}}$. Tool nose radius is $r_{\mathrm{d}}$. The diameter of the arc-shaped blade is $d_{\mathrm{d}}$. The value of $b_{\mathrm{d}}$ is determined based on the bottom width of the worm groove, as shown in Figure 3, that is usually less than the projection value of arc segment $p_{\mathrm{s} 3} \sim p_{\mathrm{s} 4}$ in the $Z$-axis.
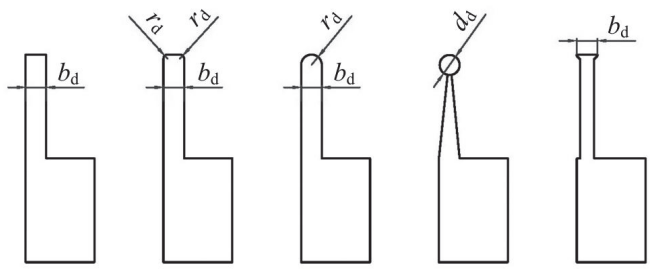

Fig. 4 Available blade shape of new roughing method

\subsection{Trapezoidal groove profile in theoretical starting position}

The new method can only ensure that the cutting point may meet the speed ratio relationship of conjugate movement. As shown in Figure 3, to process the trapezoidal groove contour $p_{\mathrm{s} 1}-p_{\mathrm{s} 3} \sim p_{\mathrm{s} 4}-p_{\mathrm{s} 2}$, we should change the positions of starting point along tooth profile in multiple feed movements. Therefore, the new method belongs to trajectory method. Because most of the material needs to be removed in roughing step, we should cut the entire tooth groove layer by layer in multiple feed movements. Trapezoidal groove profile in theoretical starting position is shown in Figure 5. Points $p_{\mathrm{s} 1} 、 p_{\mathrm{s} 2} 、 p_{\mathrm{s} 3} 、 p_{\mathrm{s} 4}$ are boundary points of tooth groove in the theoretic starting position. Any point $p_{s}$ represents the starting point of any machining cycle. Any point $p_{s}$ is located within the bounds of $p_{\mathrm{s} 1}-p_{\mathrm{s} 3} \sim p_{\mathrm{s} 4}-p_{\mathrm{s} 2}$ and on the arc $l$. Arc $l$ is the motion path of the cutting point in this machining cycle. Circle center of arc $l$ is the point $O_{0}$. The radius of arc $l$ is $r_{p}$. The radius of the worm addendum arc is $r_{a 1}$. The radius of the worm dedendum arc is $r_{f 1}$. Values of $r_{p}$ ranged from $r_{a 1}$ to $r_{f 1}$. $\theta_{r p s}$ is the acute angle between vector $O_{0} p_{s}$ and $X$-axis. When $r_{p}$ takes a constant value, according to the intersection of arc $l$ and contour line $p_{\mathrm{s} 1}-p_{\mathrm{s} 3}$, we can solve $\theta_{r \max }$. Likewise, according to the intersection of arc $l$ and contour line $p_{\mathrm{s} 2}-p_{\mathrm{s} 4}$, we can solve $\theta_{r \min }$. Obviously, values of $\theta_{r p s}$ ranged from $\theta_{r \min }$ to $\theta_{r \max }$.

When $r_{p}$ increases from $r_{a 1}$ to $r_{f 1}$ step-by-step, each step distance is just the feed amount in the radial direction. If the value of $r_{p}$ is given and fixed, the corresponding values of $\theta_{r \min }$ and $\theta_{r \max }$ can be solved easily. When $\theta_{r p s}$ decreases from $\theta_{r \max }$ to $\theta_{r \min }$ step-by-step, each step distance is just the feed amount in the circumferential direction. Controlled by variables $r_{p}$ and $\theta_{r p s}$, starting cutting points $p_{s}$ change in the above approach, that is the process of cutting groove of theoretical starting position by locus method. In fact, when solving $\theta_{r \min }$ and $\theta_{r \max }$ according to boundaries of tooth groove, the influences of blade shape, blade dimensions, and finishing allowance must be considered seriously.

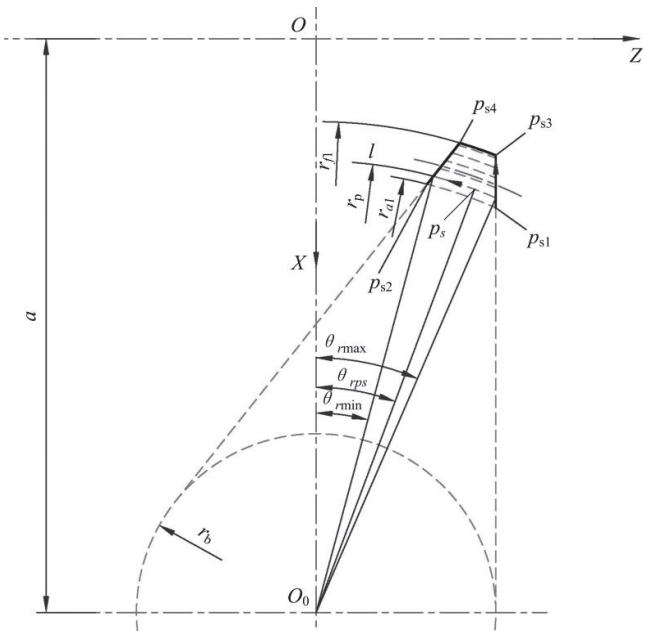

Fig. 5 Cutting groove of theoretical starting position by locus method

\subsection{Machining cycles}

When cutting entire groove by locus method, multiple machining cycles are necessary (Dong et al., 2012). The tool path for any machining cycle is shown in Figure 6 . The starting point of the cycle is point $p_{a}$. The starting point of 
cutting groove is the point $p_{s}$. According to the method of section 4.2, the value of $p_{s}$ is determined. The end point of processing groove is point $p_{b}$. The endpoint of tool retraction is the point $p_{c}$. There are four actions of the cutting tool in a cycle. They are approaching $l_{a}$, cutting $l$, retracting $l_{b}$ and returning $l_{c}$. When approaching and cutting, translation velocity of the cutting tool is specified by $F$ code. The cutting tool moves quickly during retraction and return actions.

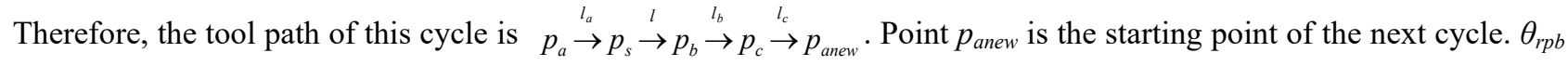
is the acute angle between vector $O_{0} p_{b}$ and $X$-axis.

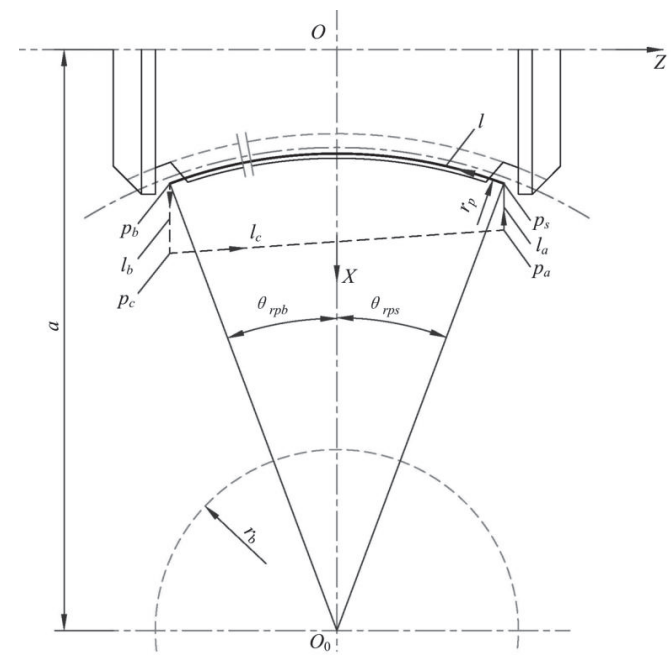

Fig. 6 Tool path of anyone machining cycle

If to be machined worm is a right-hand worm, and work piece rotates with the spindle in the clockwise direction, the coordinates of junction points can be formulated as follows:

$$
\begin{aligned}
& \left\{\begin{array}{l}
X_{p s}=2\left(a-r_{p} \times \cos \left(\theta_{r p s}\right)\right) \\
Z_{p s}=r_{p} \times \sin \left(\theta_{r p s}\right)
\end{array}\right. \\
& \left\{\begin{array}{l}
X_{p a}=X_{p s}+2 \times L_{l a} \\
Z_{p a}=Z_{p s}
\end{array}\right. \\
& \left\{\begin{array}{l}
X_{p b}=2\left(a-r_{p} \times \cos \left(\theta_{r p b}\right)\right) \\
Z_{p b}=-r_{p} \times \sin \left(\theta_{r p b}\right)
\end{array}\right. \\
& \left\{\begin{array}{l}
X_{p c}=X_{p b}+2 \times L_{l b} \\
Z_{p c}=Z_{p b}
\end{array}\right.
\end{aligned}
$$

Where, $\left[X_{p s}, Z_{p s}\right],\left[X_{p a}, Z_{p a}\right],\left[X_{p b}, Z_{p b}\right]$ and $\left[X_{p c}, Z_{p c}\right]$ are the values of junction points $p_{s}, p_{a}, p_{b}$ and $p_{c}$ in the coordinate system $X O Z$, respectively. Here, $L_{l a}$ stands for the length of $l_{a}$, and $L_{l b}$ stands for the length of $l_{b}$.

If the values of $r_{p}, \theta_{r p s}$, and $\theta_{r p b}$ are given, the corresponding coordinates of $p_{s}, p_{a}, p_{b}$ and $p_{c}$ can be obtained from Eq. (1) - Eq. (4). Therefore, the tool path of this cycle is definite. If a series of values of $r_{p}, \theta_{r p s}$, and $\theta_{r p b}$ are given according to the method of section 4.2 and 4.3 , the tool paths of multiple machining cycles are generated.

If it is a left-handed worm, work piece rotates with the spindle in the counterclockwise direction, the coordinates of junction points can also be obtained from Eq. (4).

\subsection{The same spindle phase}

To cutting ordinary cylinder screw thread by general NC lathe, threading is started at a fixed point, and thread cutting begins when the position coder mounted on the spindle outputs a 1-turn signal (Fanuc, 2004). To roughing toroidal helical groove by common NC lathe, Thread cutting is repeated the tool paths mentioned above in rough cutting. Obviously, different machining cycle has a dissimilar cutting-starting point. To avoid screw thread disorder, when start cutting point $p$ is located in the point $p_{s}$ of differing cycles, spindle must be in the same phase. Therefore, 
making good use of the 1-turn signal is very important.

In a machining cycle, the starting point of cutting action $l$ is just the ending point of approaching action $l_{a}$. If cutting a general screw thread in approaching action, and feed distance of $l_{a}$ equals an integer multiple of the thread lead, when the cutting point $p$ is located in the point $p_{s}$, spindle is in the same phase. The method can avoid screw thread disorder.

\subsection{The thread leads of different machining cycles}

In a machining cycle, cutting action $l$ is used to turn toroidal helical groove of the hourglass worm. From the machining principle of the new roughing method, work piece rotates together with the spindle at a constant rotational speed. At the same time, cutting point $p$ translates along the circular arc $l$ in XOZ. Translation velocity $F$ means feed per revolution. Values of $F$ equals the "pitch" at point $p$ on tools-gear, that is the thread lead of the machining cycle path.

From the forming principle of the Hindley worm, tooth profiles of the Hindley worm in an axial section are track lines of a generatrix. The generatrix of the Hindley worm is a straight line. Different thread leads at dissimilar cutting radius are shown in Figure 7. Where, the rotational angle of tools-gear is $\varphi_{0}$. Angular pitch of tools-gear is $\tau$. When $\varphi_{0}=0$, track line of the generatrix is straight line profile $p_{s 1}-p_{s 3}$. When $\varphi_{0}=\tau$, track line of the generatrix is straight line profile $p_{t 1}-p_{t 3}$. So on and so forth, when $\varphi_{0}=2 \tau$, track line of the generatrix is straight line profile $p_{t 5}-p_{t 7}$. Obviously, the generatrix rotates around the point $O_{0}$ in the plane $X O Z$. If points on the generatrix have a different rotation radius, they have the same angular velocity, and dissimilar linear velocity. If the rotation angle of the generatrix is a fixed value, points with distinct rotation radius have dissimilar arc length. According to this feature, linear velocity can be formulated as follows:

$$
F=\frac{2 \pi r_{p} z_{1}}{z_{2}}
$$

Where, $z_{1}$ is the number of threads of the worm. $z_{2}$ is the number of teeth of the worm gear. Thus, the linear velocity of the cutting point is translation velocity $F$, that means feed per revolution.

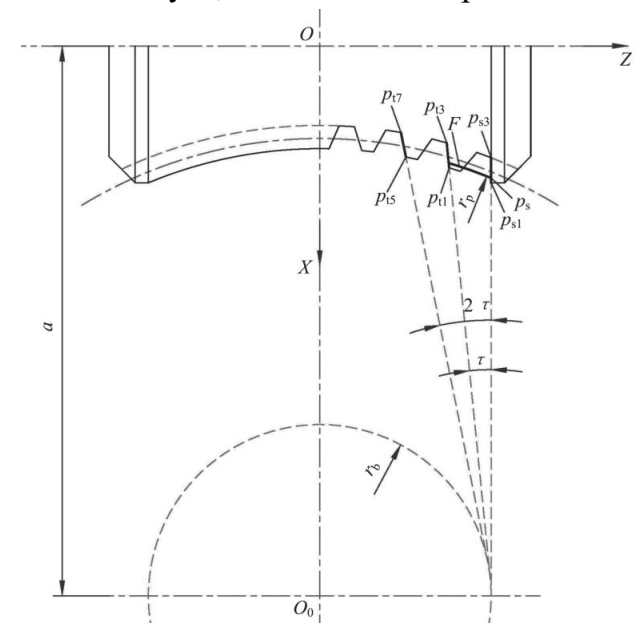

Fig. 7 Different thread leads at different cutting radius

\section{Macro programming}

When cutting tooth groove of the hourglass worm by the locus method, multiple tool paths are necessary, and control points of each path must be calculated immediately. Therefore, a user macro-program is appropriate. Based on Fanuc oi mate-tc CNC system, the macro program of the new roughing method is programmed. The flowchart is shown in Figure 8. 


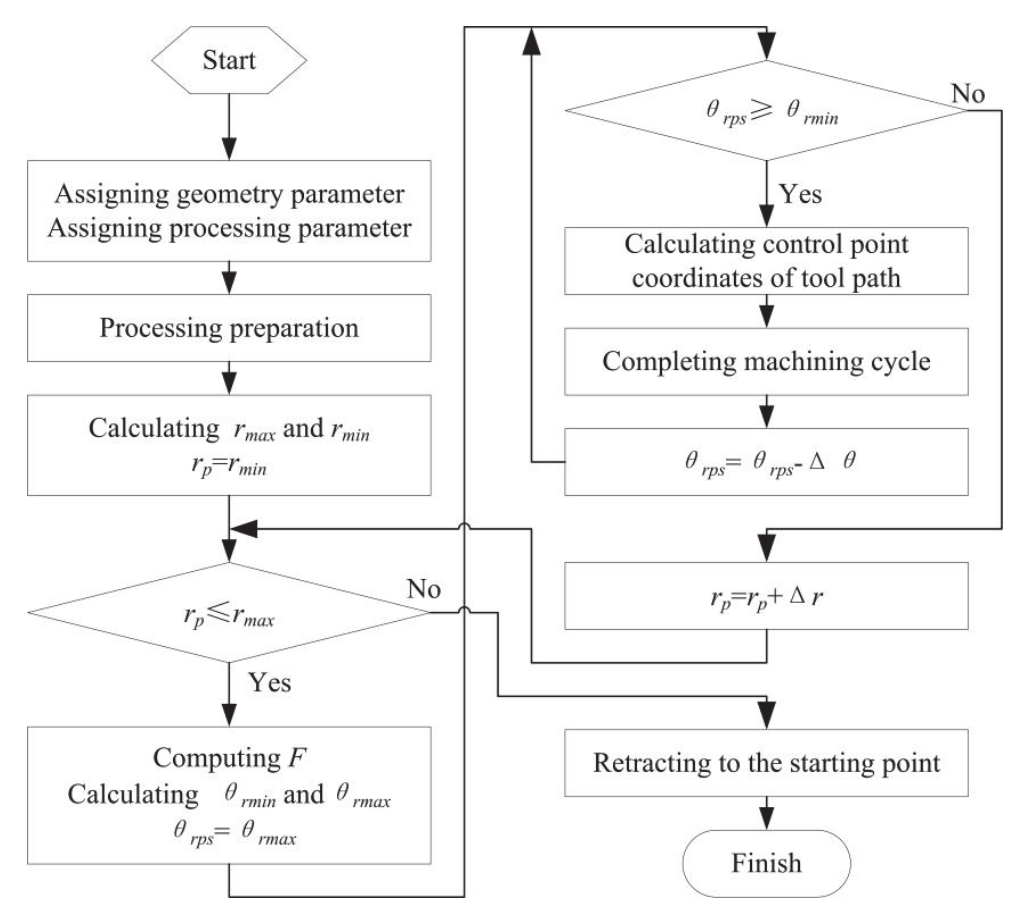

Fig. 8 The flowchart of the new method

\section{Machining example}

A right-hand hourglass worm will be described as an example of the new roughing method. The main parameters of the worm gears are given in Table 1.

Table 1 Main parameters of the worm gears

\begin{tabular}{c|c|c}
\hline \hline Item & Symbol/unit & Value \\
\hline Centre distance & $a_{12} / \mathrm{mm}$ & 125 \\
\hline Threads number of worm & $z_{1}$ & 1 \\
\hline Teeth number of worm gear & $z_{2}$ & 63 \\
\hline Transverse module of worm gear & $m_{t} / \mathrm{mm}$ & 3.3 \\
\hline Diameter of main-basic circle & $d_{b} / \mathrm{mm}$ & 77.88 \\
\hline Pitch diameter of worm & $d_{1} / \mathrm{mm}$ & 42.1 \\
\hline Addendum coefficient of worm & $h_{a 1}{ }^{*}$ & 0.7 \\
\hline Dedendum coefficient of worm & $h_{f 1}{ }^{*}$ & 0.9 \\
\hline Tooth thickness coefficient of worm & ${s_{x x}{ }^{*}}^{*}$ & 0.45 \\
\hline \hline
\end{tabular}

\subsection{Processing experiment}

A trial manufacture of the hourglass worm was carried out on a general NC lathe CK6132 with Fanuc oi Mate-TC CNC system. The process system of the trial manufacturing is shown in Figure 9. The worm blank was installed into the work spindle that rotates about axis $Z$. The work piece coordinate system was set on the rotating axis of blank, and the distance between coordinate origin and right-end was $40.0 \mathrm{~mm}$. Before roughing tooth groove, revolution surface of addendum arc had been cut out. In step 2, the cutter was mounted in the tool holder, which translating axes are axis $Z$ and $X$. The blade shape of the cutter was shown in Figure 9, and the width of the blade was $2.0 \mathrm{~mm}$. A constant rotation speed of spindle was $315.0 \mathrm{r} / \mathrm{min}$, and the step size of radial feed was $0.2 \mathrm{~mm}$. The step size of circumferential feed was also $0.2 \mathrm{~mm}$. 


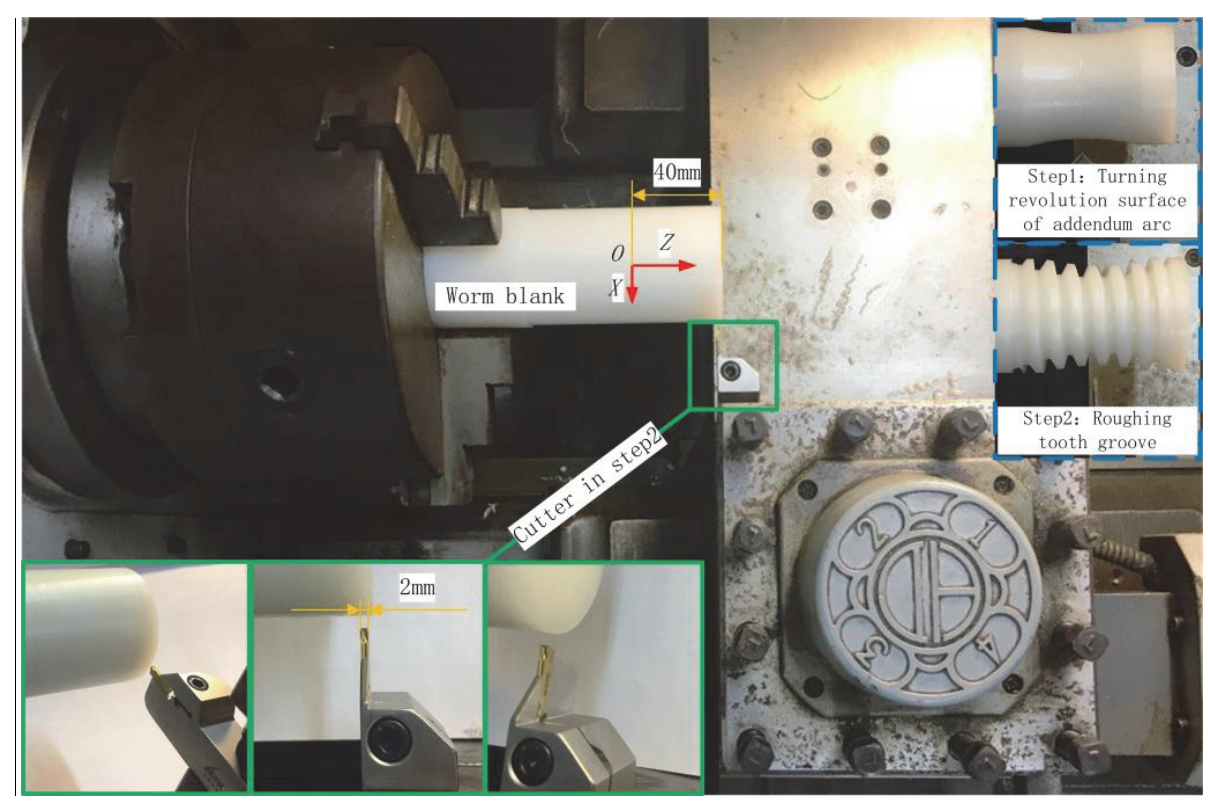

Fig.9 The process system of the trial manufacturing

Machined part of the hourglass worm with standard tooth thickness is shown in Figure 10.a. Machined part of the hourglass worm with $0.3 \mathrm{~mm}$ finishing allowance is shown in Figure 10.b.

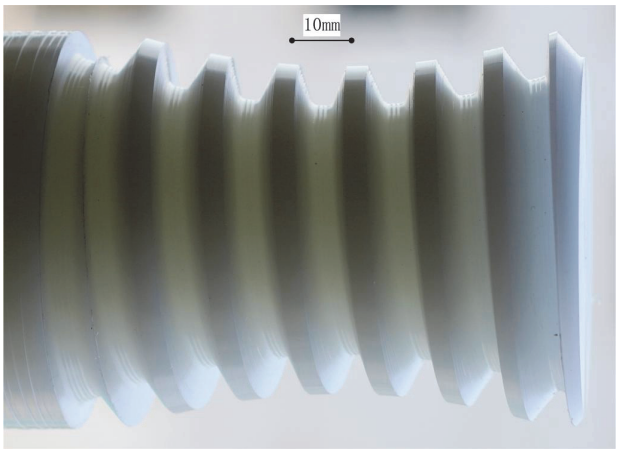

a) With standard tooth thickness

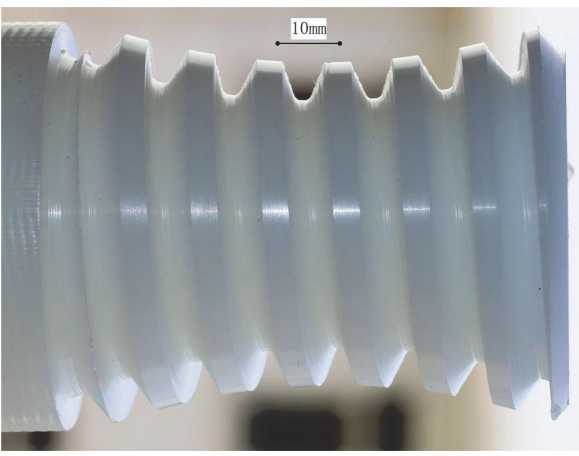

b) With $0.3 \mathrm{~mm}$ finishing allowance

Fig. 10 Machined parts of the hourglass worm

\subsection{Machining allowance}

The aim of the new method is roughing tooth groove of the hourglass worm. After machining, measurements focus on examining macroscopic dimensions of worm tooth, such as tooth profile, thread lead of worm, and so on. Measurements of machined worm tooth with standard tooth thickness are given in Table 2. The worm is shown in Figure 10.b, whose normal tooth tip thickness has been measured. The measurement is $3.26 \mathrm{~mm}$, and the theoretical value is $3.28 \mathrm{~mm}$.

Table 2 Measurements of machined worm tooth

\begin{tabular}{c|c|c|c}
\hline \hline Item & Theoretical/unit & Measurements/unit & Relative error/\% \\
\hline Normal tooth thickness of addendum & $2.68 \mathrm{~mm}$ & $2.75 \mathrm{~mm}$ & $2.61 \%$ \\
\hline Normal tooth thickness of pitch & $4.65 \mathrm{~mm}$ & $4.71 \mathrm{~mm}$ & $1.29 \%$ \\
\hline Profile angle of worm & $46.57^{\circ}$ & $45.78^{\circ}$ & $1.70 \%$ \\
\hline Thread lead of addendum & $10.14 \mathrm{~mm}$ & $10.10 \mathrm{~mm}$ & $0.39 \%$ \\
\hline \hline
\end{tabular}

The measurements are different with theoretical values, which shown in Table 2. Possible causes are as follows: 1) Tooth groove is cut out by locus method; therefore, there are residual tool marks on a machined tooth surface. 
2) When machining, spindle speed is fluctuating in a small range, thus spindle speed is not constant enough.

3) Measurement methods and operators can also cause measurement errors. However, the error of the machining worm tooth is within the tolerance of the roughing step.

In summary, the machining worm tooth with enough finishing allowance can fully meet the requirements of the roughing step.

\section{Conclusions}

The processing method of rough cutting hourglass worm tooth by two orthogonal linear axis linkage is investigated. Based on the obtained results, the following conclusions can be drawn:

(1) For roughing tooth groove of the hourglass worm, the new processing method of two orthogonal linear axis linkage is feasible and efficient. Compared with fixed-special machine tools and retrofit machine tools, general CNC lathe is more common. It's more easily to implement the new method.

(2) In one clamping to complete turning revolution surface of addendum arc and rough cutting tooth groove, general CNC lathe can achieve compound machining and automated processing. The new method has higher machining efficiency, in particular, when machining multiple threads worm.

(3) The study has some reference values to machining curve revolution surface screw threads with special tooth profile, even more, variable thread leads.

\section{Acknowledgment}

The research work in this paper was fully supported by the Fundamental Scientific Research Funds for the Central Universities of China (Grant Nos. QN2012028, QN2012030), and the Opening Research Funds for China Chongqing Key Laboratory of Time-Grating Sensing and Advanced Testing Technology (Grant No. 2013TGS003).

\section{References}

Cheng, D. X., Handbook of mechanical design (fifth edition), Volume III(2008), p.14-366, Chemical Industry Press(in Chinese).

Chen, Y., Chen, Y., Luo, W. and Zhang, G., A novel backlash-adjustable and wear-compensable hourglass worm drive: computerized design, simulation of meshing and stress analysis, Journal of Advanced Mechanical Design Systems and Manufacturing, Vol. 10, No. 2 (2016). doi:10.1299/jamdsm.2016jamdsm0029.

Dong, L., Liu, P., Wei, W., Li, H. and Dong, X., A milling method of an enveloping toroidal worm on a CNC turning center, Manufacturing Engineering and Automation Ii, Pts 1-3, Vol.482-484 (2012), pp.2188-91. doi:10.4028/www.scientific.net/AMR.482-484.2188.

Dong, L., Wang, J., et al., An NC rough turning method of an enveloping toroidal worm, Production Engineering, Vol. 6, No. 2(2012), pp.129-135.

Dudas, I., et al., Development of spiroid worm gear drive having arched profile in axial section and a new technology of spiroid worm manufacturing with lathe center displacement, International Journal of Advanced Manufacturing Technology, Vol. 79, No. 9-12 (2015), pp.1881-1892.

Du, H. J. and Jiang, Y. Y., Manufacturing processes of planar double enveloping hourglass worm drive (1988), p.21, Sichuan Publishing House of Science \& Technology(in Chinese).

Fanuc, Fanuc series oi mate-tc operator's manual(2004), No. B-64134EN/01.

Карпев(Author), А. K., Wei(Translator), R. Z., Manufacture of globoid worm drive(1958), p.72-82, China Machine Press(in Chinese).

Li, F., Long, Y., et al., A point-trajectory NC forming method and program principle for hindley worm, Machine tool \& hydraulics, Vol. 39, No. 10 (2011), pp.46-49.

Liu, C., Li, P. and Guo, X., The finite element analysis of rolling cone enveloping hourglass worm gearing based on Pro/ENGINEER, Manufacturing Engineering and Automation Ii, Pts 1-3, Vol.230-232 (2011), pp.554-556. doi:10.4028/www.scientific.net/AMR.230-232.554.

Qin, D. T. and Xie, L. Y., Modern handbook of mechanical design (2011), p.14-353, Chemical Industry Press (in Chinese).

Qiu, X. Y., Qin, D. T. and Zhang, G. H., Research on trial-manufacture of steel-steel gradual-change tooth thickness planar worm 
gear drive, Journal of Sichuan University (Engineering Science Edition), Vol. 43, No. 2 (2011), pp.222-27(in Chinese).

Rui, C., Li, H., Yang, J. and Wei, W., Research on a method for designing land surfaces of a dual-cone double enveloping hourglass worm wheel hob, Journal of Advanced Mechanical Design Systems and Manufacturing, Vol. 12, No. 4 (2018). doi:10.1299/jamdsm.2018jamdsm0090.

Shimachi, S., Gunbara, H., Kobayashi, T. and Kawada, H., Hourglass worm gears designed to concentrate surface normals, JSME International Journal Series C-Dynamics Control Robotics Design and Manufacturing, Vol. 37, No. 2 (1994), pp.347-54. doi:10.1299/jsmec1993.37.347.

Shi, W. K., Qin, D. T. and Xu, W. J., Meshing control of the double-enveloping hourglass worm gearing under the conditions of existing the errors and the load, Mechanism and MacHine Theory, Vol. 39, No. 1 (2004), pp.61-74. doi:10.1016/S0094-114X(03)00104-6.

Shi, Z., Yu, B. and He, F., Precision measurement of planar double-enveloping hourglass worms, Measurement, Vol. 91 (2016), pp.177-85. doi:10.1016/j.measurement.2016.05.021.

Wang, J. G., He, R., Deng, X. Q. and Ren, L., Manufacturing technology study on non-backlash double-roller enveloping hourglass worm, Automatic Control and Mechatronic Engineering Ii, Vol.415 (2013), pp.524-28. doi:10.4028/www.scientific.net/AMM.415.524.

Wang, S. R., Zhan, D. G., Liu, H. and Wang, S. Y., Tooth contact analysis of toroidal involute worm mating with involute helical gear, Mechanism and MacHine Theory, Vol. 37, No. 7 (2002), pp.685-91. doi:10.1016/S0094-114X(02)00003-4.

Zhang, G. H., Li, Y. Y., Hourglass worm CNC machine tools with virtual rotation center, Chinese patent disclosure ZL. 200820100105.4 (2009) (in Chinese).

Zhao, Y. P. and Zhang, Z., Mathematical model of manufacturing a novel type of hourglass worm with circular tooth profiles, Advanced Design and Manufacture Iii, Vol. 450 (2011), pp.337-40. doi:10.4028/www.scientific.net/KEM.450.337.

Zhao, Y. P., Su, D. Z., Zhang, Z., Wei, W. J. and Dong, X. Z., Mesh theory of angle modified dual tori double-enveloping toroidal worm drive, Science China-Technological Sciences, Vol. 53, No. 7 (2010), pp.1913-27. doi:10.1007/s11431-009-3156-8. 